\title{
Early Diagnosis and Treatment - The Use of Ataluren in the Effective Management of Duchenne Muscular Dystrophy
}

\author{
Eugenio Mercuri, ${ }^{1}$ Ros Quinlivan ${ }^{2}$ and Sylvie Tuffery-Giraud ${ }^{3}$ \\ 1. Catholic University, Rome, Italy; 2. Great Ormond Street Hospital and National Hospital for Neurology and Neurosurgery, London, UK; \\ 3. Laboratory of Genetics of Rare Diseases (LGMR), University of Montpellier, Montpellier, France
}

DOl: https://doi.org/10.17925/ENR.2018.13.1.31

\begin{abstract}
$\mathrm{T}$ he understanding of the natural history of Duchenne muscular dystrophy (DMD) is increasing rapidly and new treatments are emerging that have the potential to substantially improve the prognosis for patients with this disabling and life-shortening disease. For many, however, there is a long delay between the appearance of symptoms and DMD diagnosis, which reduces the possibility of successful treatment. DMD results from mutations in the large dystrophin gene of which one-third are de novo mutations and two-thirds are inherited from a female carrier. Roughly $75 \%$ of mutations are large rearrangements and $25 \%$ are point mutations. Certain deletions and nonsense mutations can be treated whereas many other mutations cannot currently be treated. This emphasises the need for early genetic testing to identify the mutation, guide treatment and inform genetic counselling. Treatments for DMD include corticosteroids and more recently, ataluren has been approved in Europe, the first disease-modifying therapy for treating DMD caused by nonsense mutations. The use of ataluren in DMD is supported by positive results from phase IIb and phase III studies in which the treatment produced marked improvements in the 6-minute walk test, timed function tests such as the $10 \mathrm{~m}$ walk/run test and the 4-stair ascent/descent test compared with placebo. In these trials, ataluren was well tolerated and adverse event profiles were similar to placebo. As such disease-modifying treatments become more widely available, the outlook for children with DMD will improve but physicians must be aware of the disease, rapidly initiate testing where it is suspected and promptly begin appropriate treatment.
\end{abstract}

\section{Keywords}

Duchenne muscular dystrophy, early diagnosis, nonsense mutations, treatment, disease-modifying therapy, ataluren

Disclosure: Eugenio Mercuri has received consulting fees from PTC Therapeutics, Sarepta, BioMarin, Roche and Biogen. Ros Quinlivan has received honoraria for consultation, lectures and organisation of teaching symposia from PTC Therapeutics, Genzyme, Santhera, Roche, Ultragenyx (Biomarin) and Novartis. Sylvie Tuffery-Giraud has served on a scientific advisory board for PTC Therapeutics in France and remuneration for this activity was paid to the University of Montpellier. The University of Montpellier also received speaker honoraria from PTC Therapeutics. Review Process: Double-blind peer review

Compliance with Ethics: Written informed consent was not obtained from the patient case included in this article: no identifying information or images have been used. Acknowledgements: Editorial assistance was provided by James Gilbart of Touch Medical Media Authorship: All named authors meet the criteria of the International Committee of Medical Journal Editors for authorship for this manuscript, take responsibility for the integrity of the work as a whole and have given final approval for the version to be published.

Open Access: This article is published under the Creative Commons Attribution Noncommercial License, which permits any non-commercial use, distribution, adaptation, and reproduction provided the original authors and source are given appropriate credit. (c) The Authors 2018.

Received: 7 November 2017

Accepted: 5 February 2018

Citation: European Neurological Review. 2018;13(1):31-7

Corresponding Author: Eugenio Mercuri, Paediatric Neurology, Policlinico Gemelli, Largo Gemelli, 00168, Rome, Italy. E: eumercuri@gmail.com

Support: This article was supported by PTC Therapeutics International Ltd. The views and opinions expressed in the article are those of the authors and not necessarily those of PTC Therapeutics International Ltd.
These are exciting times in Duchenne muscular dystrophy (DMD); the first disease-modifying drug for this indication has been approved in the European Union for ambulatory DMD in patients $>5$ years; other drug treatments are in the pipeline. ${ }^{1-5}$ These treatments can be most impactful when used early in the disease process and it is therefore imperative that possible cases of DMD are recognised early, referred to specialist centres promptly and are diagnosed and treated urgently. This is a challenge for neurologists, especially as diagnosis is often delayed with a consequently poorer prognosis. ${ }^{6,7}$ This review therefore discusses timely issues of current and emerging approaches to the diagnosis and treatment of DMD. This includes up-to-date knowledge of the natural history and diagnosis of DMD, an instructive case example and the current understanding of the genetics of DMD. It also evaluates the clinical study evidence supporting the use of ataluren (Translarna' ${ }^{\text {TM }}$, PTC Therapeutics) in nonsense mutation DMD (nmDMD).

\section{A typical journey in Duchenne muscular dystrophy - from diagnosis to referral, treatment and later development}

DMD is an X-linked recessive disorder that affects 1:3,500-1:5,000 live male births. ${ }^{.-10}$ The journey of a child with DMD typically starts with delayed walking (later than 18 months) accompanied with developmental and speech delay, possible autism/behavioural problems, cognitive impairment, worsening motor skills, toe walking, falls, calf hypertrophy, a positive Gowers' sign and significantly increased serum creatinine kinase levels (often $>10,000 \mathrm{IU} / \mathrm{L}$ ). 5,11,12 Although the characteristic presentation is impaired walking, other signs should also be looked for and recognised at an earlier stage. Diagnostic procedures can still involve a muscle biopsy but genetic testing should be the gold standard since it can inform therapy. Genetic testing involves multiplex ligation-dependent probe amplification (MLPA) for detecting deletions or duplications and full gene sequencing for detecting small point mutations including nonsense mutations. ${ }^{1,13}$ Delayed diagnosis can mean a window of opportunity for treatment is missed with consequent irreversible worsening of muscle function and other effects. ${ }^{6,7,14,15}$

Until recent years, the journey of a child with DMD ended with early death during the teenage years. In the 1960s, mean life expectancy was as short as 14.4 years; prior to 1990, range of life expectancy for boys with DMD was 16-21 years (mean 19 years). Since then, developments, particularly the availability of home ventilation, have raised life expectancy to 25-35 years (mean 28 years) and, on occasion, patients survive beyond the age of 40 years. ${ }^{16,17}$ 
The DMD journey can be substantially improved with early diagnosis and good disease management. This has been assisted by the development of agreed international standards of care which specify an optimal process that involves a multidisciplinary approach and recommends the use of treatments including corticosteroids. ${ }^{8,18} \mathrm{~A}$ recent meta-analysis that included 12 DMD studies with a total of 667 participants found evidence that muscle strength was improved for up to 2 years with corticosteroid treatment. ${ }^{19}$ In DMD, corticosteroids provide various benefits including: increased muscle strength, prolonged ambulation, reduced need for scoliosis surgery and preserved respiratory and cardiac function in adults. ${ }^{19-24}$ Corticosteroids can be used in children from 4 years of age provided they have immunity to chickenpox. These treatments, however, are associated with various risks that include weight gain, Cushingoid features, behaviour changes, growth delay, fractures, cataracts and skin changes. ${ }^{25}$ The adverse effect profiles vary somewhat between different corticosteroids. These risks, however, appear to be outweighed by the benefits as illustrated by data from a population-based study of 477 eligible DMD cases identified by the Muscular Dystrophy Surveillance Tracking and Research Network. ${ }^{26}$ Loss of ambulation (LOA) in untreated patients ( $n=162$ ) occurred at an average age of 10.3 years whereas in patients who received long-term corticosteroid treatment $(n=78)$, LOA occurred 2 years later at a mean age of 12.3 years $(p<0.05)$.

In $\mathrm{DMD}$, the patient journey frequently leads to cardiomyopathy which is an increasingly important cause of left ventricular (LV) dysfunction resulting in arrhythmias and heart failure. ${ }^{27}$ Current recommendations advise using angiotensin converting enzyme (ACE) inhibitors after the development of LV dysfunction to improve cardiac function. ${ }^{18}$ More recently, several studies have shown that the onset of LV dysfunction can be delayed and mortality decreased with the early commencement of ACE inhibitors by the age of 10 years. ${ }^{28,29}$ In patients who cannot tolerate ACE inhibitors, angiotensin receptor blockers can be used as an alternative. ${ }^{30}$

The case example below illustrates the journey of one patient with DMD and emphasises the serious effects the disease has on the normal development of a young boy. However, it also indicates that the use of a treatment that addresses the cause of DMD (ataluren) can prolong ambulation beyond the expected age and delay other disease milestones. When used in combination with other standard-of-care therapies such as a corticosteroid and physiotherapy, this treatment has the potential to improve quality of life and prognosis in DMD.

Final survival outcomes with corticosteroid treatment are not yet known and greater long-term experience of these drugs is needed. Nevertheless, there is a marked improvement in boys who receive them. ${ }^{20,22,23,31}$ In the adult practice at the National Hospital for Neurology and Neurosurgery, London, UK, among a cohort of 54 adults with DMD and receiving corticosteroids, only one required non-invasive ventilation at the time of transfer to adult services compared with 22/54 who were not receiving corticosteroids. The importance of corticosteroid treatment in DMD was further emphasised by the Food and Drug Administration (FDA) approval in 2017 of deflazacort for the treatment of this disease in children aged 5 years and older. ${ }^{25,32,33}$ Recent work has shown that longterm glucocorticoid therapy in DMD cuts death risk by $50 \% .^{25}$ It is likely in the near future that all alternative strategies will need to be proved as complementary to this treatment. Such treatments however need to be used cautiously; a recent study investigated associations between timing of corticosteroid treatment initiation and clinical outcomes in DMD and notably found that an earlier start of corticosteroids is potentially linked to earlier heart disease..$^{33}$
Case example - Boy with DMD, aged 13 years 8 months, referred for management

A boy aged 18 months presented with motor delay, was not yet walking and had creatine kinase levels of 9,249 IU/L. Muscle biopsy showed absent dystrophin staining. MLPA showed no deletion or duplication. Full DNA sequencing on muscle tissue showed a nonsense mutation (c.8069T $>\mathrm{G}$ ) in exon 55 resulting in a change of an amino acid to a premature stop codon $\mathrm{p}$.(Leu2690*) in the dystrophin protein that leads to premature termination of protein translation.

At age 6 years, he commenced daily prednisolone $(15 \mathrm{mg}$ daily, $0.75 \mathrm{mg} / \mathrm{kg} /$ day) and vitamin D $400 \mathrm{IU} /$ day. At age 9 years, he was recruited into the phase $\mathrm{\| b} 007$ ataluren study and randomised to receive ataluren $80 \mathrm{mg} / \mathrm{kg} /$ day. Some months into the trial, he fell and sustained a spiral fracture of the right femur but made a full recovery following intensive physiotherapy. Echocardiography 12 months before referral (age 12 years) was reported as normal.

At referral, aged 13 years and 8 months, he was receiving prednisolone $15 \mathrm{mg}$ daily $(0.4 \mathrm{mg} / \mathrm{kg} / \mathrm{day})$. He was ambulant with a waddling gait and had a lumbar lordosis with positive Gowers' sign. He was prepubertal with a short stature, had mild Cushingoid features, marked calf hypertrophy, tightness of the Achilles tendon and proximal muscle weakness. He had no symptoms of nocturnal hypoventilation, no muscle pains/cramps or myoglobinuria. Echocardiography revealed left ventricular fractional shortening (23-27\%) and mild left ventricular hypokinesia/dyskinesia and he was consequently commenced on perindopril $2 \mathrm{mg}$ daily.

He was started on ataluren 40 mg/kg/day (375 mg morning, 375 mg noon and $750 \mathrm{mg}$ bedtime). He is now aged over 16 years and is receiving an increased ataluren dose in line with his current weight (500 mg/500 mg/875 mg doses each day). His 6-minute walking distance decreased from $315 \mathrm{~m}$ at age 13 years and 8 months to $166 \mathrm{~m}$ at age 16 years and 8 months, nonetheless he remains ambulant at this age in contrast to natural history data for boys with DMD that indicates the average age for loss of ambulation is 12.3 years. His North Star Ambulatory Assessment score decreased from 15 to 4 over the same period. He was also receiving testosterone for delayed puberty, vitamin D (increased to 20,000 IU every 2 weeks) and low dose prednisolone $15 \mathrm{mg} /$ day. Repeated echocardiography showed normal cardiac function. Dual-energy X-ray absorptiometry showed bone density was satisfactory. Annual blood testing (regular monitoring with ataluren treatment) showed that cholesterol, triglycerides, urea and electrolytes, liver function, full blood counts and cystatin $\mathrm{C}$ remain stable. Ataluren was well tolerated with no adverse effects.

The optimal treatment schedule for corticosteroids in DMD is unclear and it is often a matter of balancing efficacy with side effects. These issues are being investigated further in the ongoing Finding the Optimum Regimen for DMD (FOR-DMD) study (NCT01603407, n=225) that aims to establish whether daily/intermittent prednisolone or daily deflazacort provides optimum treatment.

In the DMD journey, LOA probably correlates with long-term survival, so it is likely that increasing LOA age will, in turn, improve survival. 20,22,23,3,34 There may also be an additive effect when corticosteroids are used in combination with a disease-modifying drug such as ataluren. ${ }^{35}$ Given this experience, when seeing a new patient with DMD it is advisable to prepare both the boy and his family to expect survival into adulthood. 
The natural history of DMD and the journey experienced by children with the disease therefore is changing and will continue to change as new treatment options emerge. Improvements in prognosis can be achieved with early diagnosis that enables the optimum effect of treatments such as corticosteroids and ataluren. It is also vital that patients with nonsense mutations are identified indicating those who are eligible for ataluren treatment.

\section{Genetic testing - a crucial component in accurate diagnosis of Duchenne muscular dystrophy}

DMD results from a deletion, duplication or point mutation (nonsense, frameshifting, etc.) in the dystrophin gene at Xp21 and is recognisable by an absence or reduced levels of dystrophin staining in muscle biopsies. ${ }^{15,8,13}$ The gene encoding dystrophin is located on the $X$ chromosome (Xp21.2), is very large (2.2 Mb) and exhibits a high mutation rate. ${ }^{36}$ This gene has very long introns and the coding sequence consists of 79 exons involving seven promoters (three full length and four internal), which give rise to different protein isoforms. In skeletal muscle, the full-length protein is $427 \mathrm{kDa}$, is found at the sarcolemma and, through binding to transmembrane proteins, connects to the extracellular matrix. Dystrophin has a structural role providing strength, flexibility and stability to muscle fibres and is involved in the regulation of signalling processes. ${ }^{36,37}$

Dystrophinopathies are allelic conditions linked to mutations in the DMD gene that codes for the dystrophin protein. There is a wide spectrum of disease severity ranging from paucisymptomatic forms (cramps and myoglobinuria) and X-linked dilated cardiomyopathy through to Becker muscular dystrophy (BMD) to DMD. Disease severity is dependent on the amount and functionality of the dystrophin protein present. ${ }^{38,39}$ In approximately $80 \%$ of cases there are large rearrangements consisting of deletions or duplications of exon(s); the remaining $20 \%$ consist of point mutations. .0-2 $^{2}$

The reading frame rule, which was described over 25 years ago, largely explains how different mutations within the dystrophin gene could give rise to different clinical presentations. ${ }^{43}$ Mutations that introduce a single (due to a nonsense mutation) or a series of premature stop codons (due to a frameshifting mutation) in the coding sequence of DMD mRNA lead to the degradation of the mutant transcript by the mRNA surveillance mechanisms and/or premature termination of translation. As a result, no dystrophin protein is synthesised most often leading to the most severe disease, DMD. In contrast, mutations that preserve an open reading frame allow the synthesis of a shorter but partially functional dystrophin protein and are associated with a BMD phenotype of variable severity depending on the amount and functionality of the mutant dystrophin. The reading frame rule holds true for $92-6 \%$ and $93 \%$ of the mutations in DMD and BMD patients respectively. ${ }^{41,42}$

The strategy of genetic testing in DMD is to look for the most common mutations first. Deletions are present in about $67-72 \%$ of cases and duplications in $7-11 \%$ of cases. They are not randomly distributed but rather clustered in 'hot spots' around exons 45-55 and exons 2-20, respectively. ${ }^{141,44-46}$ The multiplex polymerase chain reaction assay has been widely used for a long time as it is a simple and inexpensive procedure that detects $98 \%$ of deletions. ${ }^{47}$ This, however, does not detect duplications nor allow definition of the exact boundary of the deletions in all cases.

Currently, detection of large rearrangements in the DMD gene relies on gene dosage techniques in both male patients and female relatives. These include MLPA using exonic probes on 79 exons, array comparative genomic hybridization (aCGH) using over 20,000 probes covering the entire gene (introns and exons) and massively parallel next generation sequencing (NGS), which can cover 79 exons with intronic boundaries or the entire gene ${ }^{1,48-53}$ and can readily identify deletions and duplications. ${ }^{52}$ These last few years, MLPA has become the most common dosage technique used. It is highly efficient but false positive results are possible where there is a single exon deletion caused by a point mutation or a single nucleotide polymorphism on the probe-binding region. As recommended by the laboratory best practice guidelines, an independent method should always be used to verify the presence of a single exon.

In addition, false negatives are possible when the deletion is not covered by the probe hybridisation or can give wrong information regarding the extent of the deletion. ${ }^{54,55}$ Using aCGH has contributed to the characterisation of the full spectrum of large rearrangements in the DMD gene that include triplications and complex rearrangements (non-continuous rearrangements) and allowed the precise definition of intronic boundaries. ${ }^{48}$ The gene dosage techniques, however, cannot define the location or orientation of duplications and triplications.

In $\mathrm{DMD}$, about $20 \%$ of mutations are point mutations, of which almost half (48\%) are nonsense mutations (10\% of all mutations). ${ }^{41,42}$ In contrast to large rearrangements, these point mutations are randomly located and their identification requires entire gene sequencing using either an exon-by-exon (Sanger) approach or using the newer NGS technique. In some rare cases, nonsense mutations can occur in BMD. They are preferentially found in the block of in-frame exons 25-40, where the mutations have been shown to induce some degree of exon skipping. ${ }^{56-59}$ This can eliminate a nonsense mutation giving rise to an in-frame and translatable transcript and a limited amount of internally truncated and partly functional dystrophin. It is therefore important to check the disease phenotype and perform dystrophin analysis in addition to genetic analyses when nonsense mutations are found in this region of the DMD gene. ${ }^{00}$

Given the value of information provided by genetic analyses, muscle biopsy now is only recommended when genetic analysis is inconclusive (however, some laboratories may request confirmation of a dystrophinopathy diagnosis before engaging in sequencing analyses for identification of point mutations). In such unclear cases, RNA sequencing analysis may also be tried to reveal deep intronic mutations. ${ }^{61}$ RNA and protein studies are also useful to explore further some cases of discordance between genotype and phenotype..$^{00}$ These approaches are described in the Best Practice Guidelines on Molecular Diagnostics in DMD/BMD, which were published in 2010.62

Genetic testing is important to confirm a diagnosis and to justify multidisciplinary care and early corticosteroid treatment for patients. Establishing a mutation-specific diagnosis is also important in determining whether it is a de novo mutation (in about one-third of isolated cases) or inherited through a maternal carrier (two-thirds of cases). This allows for appropriate genetic counselling of family members and can enable prenatal diagnosis and preimplantation genetic diagnosis (in in vitro fertilisation [IVF]) in female carriers. Genetic testing also directs treatment since some therapies are suitable only for certain types of mutations. ${ }^{13,63}$ Genetic testing is therefore a critical tool in the accurate diagnosis of DMD and helps avoid missing the opportunity for personalised treatment.

Genetic testing occurs only after DMD is suspected and the patient is referred to a specialist centre. One study in the UK showed that symptoms were first reported at a mean age of 2.7 years but a creatine 
kinase (CK) test was not performed until a mean age of 4.2 years and genetic analysis at 4.3 years when there was no family history. ${ }^{15}$ It is critical that this delay is reduced; symptoms of DMD need to be recognised more quickly so that genetic testing can be conducted as early as possible to identify the mutation, thus enabling access to standards of care and appropriate treatment to be initiated. Deletions constitute about $55 \%$ of DMD cases for which antisense-mediated exon-skipping treatments to restore an open reading frame and a shorter partially functional protein are applicable. ${ }^{41}$ Nonsense mutations constitute 10\% of DMD cases for which treatments enabling readthrough of stop codons and a full-length potentially fully functional protein are applicable. ${ }^{41}$

In DMD epidemiology and management, patient registries are highly valuable and are helpful in identifying suitable candidates for clinical trials. An example is the Universal Mutation Database-Duchenne Muscular Dystrophy (UMD-DMD) in France that aims to include all French patients with a genetically confirmed diagnosis of dystrophinopathy (i.e. DMD gene mutation identified) through the contribution of a network of laboratories and clinical reference centres. ${ }^{42}$ This is a searchable database that now includes data on 2,898 patients and unpublished data. In this registry, $61 \%$ of dystrophinopathy patients have DMD and among mutations, $79 \%$ are large rearrangements and $21 \%$ are point mutations. Of all mutations, $10 \%$ are nonsense mutations.

In DMD management therefore, it is critically important to be aware that disease caused by certain deletions and nonsense mutations can be treated but is essential to identify which is present in each new patient to guide their type of treatment (the exon skipping therapy, eteplirsen has been approved by the FDA and is now available in the US). ${ }^{64}$ This mutation data enables well-informed genetic counselling of all families involved. In some territories, there is a long delay between symptom reporting and DMD diagnosis - this must be shortened to facilitate access to standards of care and to enable successful treatment as early as possible. There are some treatments in development that are not restricted to patients with specific mutations but can benefit to all patients with DMD as they address the primary defect (gene therapy, utrophin modulation). ${ }^{65}$ Some ongoing clinical trials conducted by Summit Therapeutics (NCT02858362), Pfizer (NCT03362502) and Solid Biosciences, LLC (NCT03368742) are investigating these approaches. For these strategies testing is not essential but genetic testing is still mandatory to ascertain the diagnosis of DMD before the inclusion of a patient in a clinical trial.

\section{Recent data supporting the first disease- modifying treatment in Duchenne muscular dystrophy (ataluren)}

When conducting clinical trials in DMD it is important to select endpoints that are appropriate to the treatment under investigation, its mechanism of action and treatment duration. The most common endpoint used in DMD is the 6-minute walk test (6MWT). ${ }^{66}$ Timed function tests (TFTs) including the $10 \mathrm{~m}$ walk/run test, 4-stair ascent or descent and time to $10 \%$ persistent worsening of 6MWT are also used. There is, however, a need for wider ranging assessments in DMD; no single outcome measure in DMD is ideal for all stages of the disease. A wider measure of physical capability is provided by the North Star Ambulatory Assessment (NSAA), which is a tool specifically developed for DMD patients. ${ }^{67,68}$ Combinations of these endpoints have been used in the clinical trials of ataluren, drisapersen, tadalafil, eteplirsen and anti-myostatin monoclonal antibody for the treatment of DMD. ${ }^{35,69-73}$ The inclusion criteria of clinical studies for these drugs in DMD have included specific lower limits for baseline 6-minute walk distance (6MWD) ranging from $\geq 75 \mathrm{~m}$ to $\geq 300 \mathrm{~m}^{35,74}$ and some had a specific range (e.g. 200-400 $\mathrm{m}$ for tadalafil). ${ }^{75}$
Figure 1: Baseline 6-minute walking distance as a key factor predicting decline in Duchenne muscular dystrophy

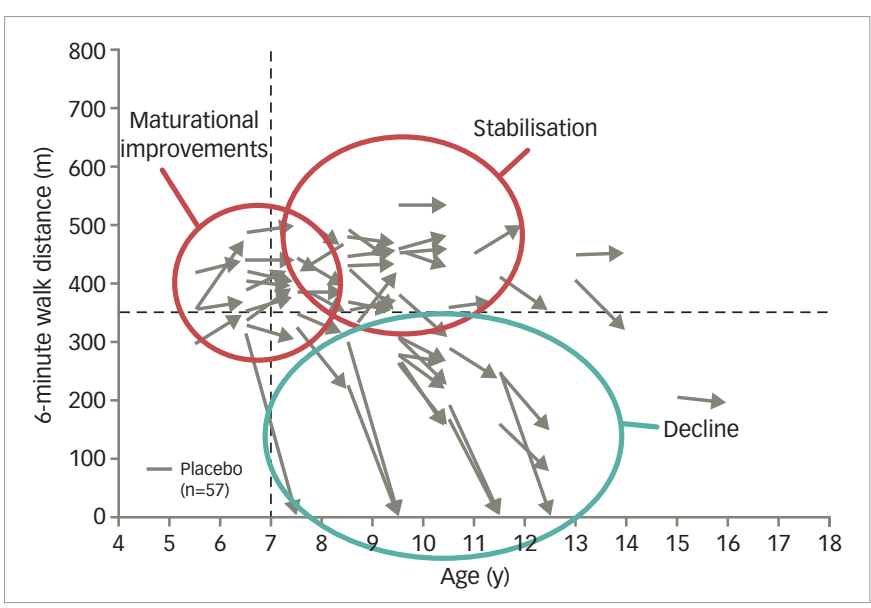

Reproduced with permission from McDonald et al., 2013. ${ }^{76}$

In DMD studies, shorter baseline 6MWDs are associated with more rapid disease progression and functional outcome measures are less sensitive in these more advanced cases. ${ }^{76}$ It is therefore an important criterion in selecting clinical study participants. This was highlighted in one study $(n=57)$ in which children with longer 6MWDs at baseline $(\geq 350 \mathrm{~m})$ showed a mean decrease of $5 \mathrm{~m}$ over 48 months, whereas children with shorter 6MWD at baseline (<350 m) showed a much greater decrease of $107 \mathrm{~m}$ (Figure 1). ${ }^{76}$ Selecting children with baseline 6MWDs in the range of 300$400 \mathrm{~m}$ may be the optimal subgroup to show a treatment effect in a 1-year clinical trial in DMD; the difference between active treatment and placebo will be most marked in this group. Alternatively, other measures may be used in combination with baseline 6MWD to define the patient population; for example, stand from supine $>5$ seconds corresponds approximately to $6 \mathrm{MWD}>400 \mathrm{~m}$ and may be used as a threshold instead. In addition, patients with a baseline 6MWD <300 m have a higher muscular fat fraction as seen on magnetic resonance spectrometry (MRS) than those with a baseline $6 \mathrm{MWD}>350 \mathrm{~m}$. A >0.8\% fat fraction in patients' muscles correlates with a sharp loss of their ability to walk.77

Ataluren is currently the only disease-modifying drug that is approved in Europe for use in nmDMD in ambulatory boys aged $>5$ years. Ataluren has recently been recommended for reimbursement by the UK National Institute for Health and Clinical Care Excellence (NICE) and by the Agenzia Italiana del Farmaco (AIFA). ${ }^{78-80}$

Ataluren has been shown to bind to the ribosome and enables readthrough of a premature stop codon caused by a nonsense mutation

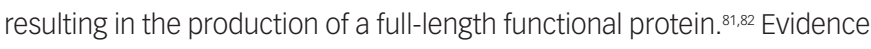
supporting the use of ataluren in DMD comes from several clinical trials including a phase IIb study in ambulatory patients $(n=174)$ in which males $\geq 5$ years with DMD nonsense mutations and a baseline $6 \mathrm{MWD} \geq 75 \mathrm{~m}$ were randomised (1:1:1) to ataluren $80 \mathrm{mg} / \mathrm{kg} /$ day, or $40 \mathrm{mg} / \mathrm{kg} /$ day or placebo over 48 weeks followed by an openlabel extension (70\% of patients in all three groups were receiving corticosteroids). After 48 weeks, patients receiving ataluren $40 \mathrm{mg} / \mathrm{kg} /$ day showed a $31.7 \mathrm{~m}$ smaller reduction in 6MWD compared with placebo (adjusted $\mathrm{p}=0.0367$ ) (Figure 2). ${ }^{35,83}$ These findings were strengthened by clinically meaningful differences of 1.5 seconds in three of four timed function tests (worsening of $10 \mathrm{~m}$ walk/run, 4-stair ascent and 4-stair descent times) for ataluren $40 \mathrm{mg} / \mathrm{kg} /$ day compared with placebo. Further evidence came from a post-hoc subgroup analysis 
Figure 2: Effects of ataluren on walking ability (6-minute walking distance) versus placebo over 48 weeks in the phase Ilb study

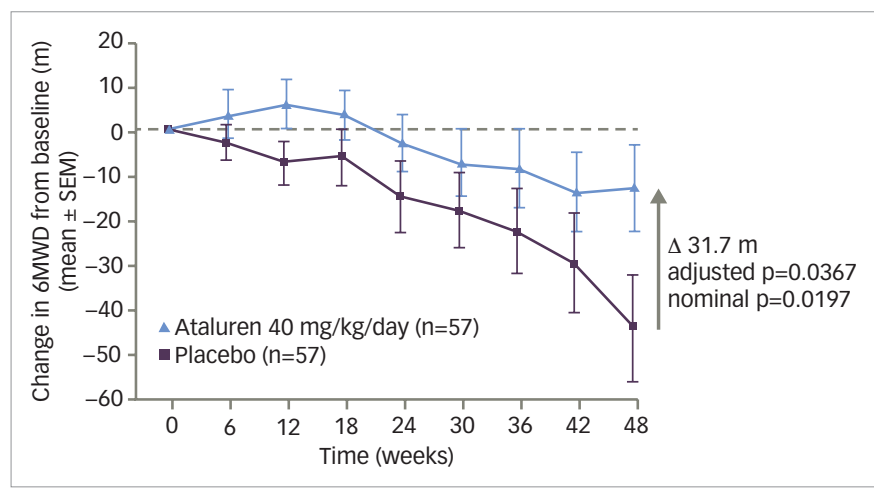

$6 M W D=6$-minute walk distance; SEM = standard error of mean.

Reproduced with permission from Bushby et al., 2014. ${ }^{35}$

Figure 3: Pre-specified analysis of change in 6-minute walking distance in a subgroup of boys with baseline values of $\geq 300-<400 \mathrm{~m}$ in the phase III ataluren confirmatory trial for Duchenne muscular dystrophy

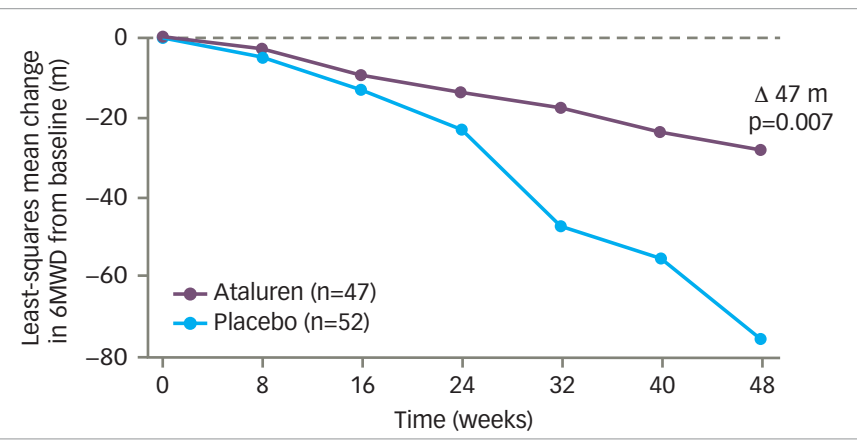

6MWD = 6-minute walk distance. Two patients (one per arm) were excluded because they did not have a Duchenne muscular dystrophy nonsense mutation.

Reproduced with permission from McDonald CM et al., 2017. ${ }^{86}$

of 'decline phase' children aged 7-16 years $(n=61)$ who had a baseline $6 \mathrm{MWD}$ between $\geq 150$ and $\leq 80 \%$ predicted for their age and height and were taking corticosteroids. This subgroup showed a larger $49.9 \mathrm{~m}$ reduction in $6 \mathrm{MWD}$ decline for ataluren $40 \mathrm{mg} / \mathrm{kg} /$ day compared with placebo (nominal $\mathrm{p}=0.0096$ ). ${ }^{35}$ Importantly, there were no safety issues with ataluren; no patients discontinued treatment due to adverse events and their incidence and types were similar for both drug doses and for placebo.

Even though the phase IIb study did not meet its primary endpoint, based on the consistent trends seen in the primary (6MWD), secondary (TFTS) and tertiary (myometry, quality of life, falls, step activity and wheelchair use) endpoint results of this study, ataluren was approved by the European Commission in July 2014 and the justification for this decision was given by Hass et al. in a European Medicines Agency review. ${ }^{83}$ In September 2017 however, the FDA opined that evidence in support of the efficacy of ataluren in DMD was inconclusive and that further study would be necessary. ${ }^{84}$ This was contrary to the European decision. The FDA decision is being appealed and a decision is pending as of January 2018.

The phase III ataluren confirmatory trial for DMD (ACT-DMD) $(n=230)$ recruited males with nonsense mutations who were aged $\geq 7$ and $\leq 16$ years, were receiving corticosteroids $\geq 6$ months and had a baseline $6 \mathrm{MWD} \geq 150 \mathrm{~m}$ and $\leq 80 \%$ of that predicted for age and
Table 1: Secondary endpoints - time function test differences between active treatment and placebo in two populations in the phase III ataluren confirmatory trial in Duchenne muscular dystrophy

\begin{tabular}{|c|l|l|l|}
\hline \multicolumn{1}{|c|}{$10 \mathrm{~m}$ walk/run } & 4 -stair ascent & 4-stair descent \\
\hline $\begin{array}{c}\text { ITT population } \\
\text { Change (seconds) }\end{array}$ & -1.2 & -1.8 & -1.8 \\
\hline p-value (ataluren vs placebo) & 0.117 & 0.058 & 0.012 \\
\hline $\begin{array}{c}\text { Pre-specified } \geq 300 \text { to }<400 \mathrm{~m} \\
\text { baseline 6MWD subgroup } \\
\text { Change (seconds) }\end{array}$ & -2.1 & -3.6 & -4.3 \\
\hline \begin{tabular}{c} 
p-value (ataluren vs placebo) \\
\hline
\end{tabular} & 0.066 & 0.003 & $<0.001$ \\
\hline
\end{tabular}

6MWD = 6-minute walk distance; $I T T=$ intent to treat. Data sourced from McDonald et al., 2016. ${ }^{85}$

height.85,86 Patients were randomized (1:1) to ataluren $40 \mathrm{mg} / \mathrm{kg} / \mathrm{day}$ or placebo. In the intention to treat (ITT) population at 48 weeks, patients receiving ataluren had a smaller decrease in 6MWD compared with patients receiving placebo but this was not significant $(p=0.213)$. In a pre-specified analysis of boys whose 6MWD was $\geq 300-<400$ $m$ at baseline $(n=99)$, patients on ataluren had a $42.9 \pm 15.9 \mathrm{~m}$ (least squares mean \pm standard error) smaller decrease in their 6MWD compared with patients on placebo ( $\mathrm{p}=0.007$ ) (Figure3). Secondary efficacy endpoints (10 m run/walk, 4-stair ascent/descent) showed advantages for ataluren treatment versus placebo in the ITT population and even greater differences in the baseline $6 \mathrm{MWD} \geq 300-<400 \mathrm{~m}$ subgroup (Table 1). For the ITT population at 48 weeks, there was a numerical but non-significant treatment difference of 0.8 points $(p=0.128$ ) in the total NSAA scores which favoured ataluren treatment. When this score was linear transformed, the difference was 1.5 points which remained non-significant $(p=0.268)$. In the baseline $6 \mathrm{MWD} \geq 300-<400 \mathrm{~m}$ subgroup, however, the treatment effect was more evident with a total NSAA score difference of 1.7 points $(p=0.037)$ and a linear transformed score difference of 4.3 points $(\mathrm{p}=0.041)$. In a post-hoc analysis of this study, TFT scores were significantly different between ataluren and placebo for both entire ITT group and 300-400 m subgroup. For the ITT population, composite TFT was $-1.6 \pm 0.7(p=0.023)$. For $300-400 \mathrm{~m}$ subgroup, composite TFT was $-3.5 \pm 1.0(p=0.0007)^{86}$

In the ACT-DMD study, safety findings were consistent with the phase IIb results. The incidence and types of adverse events reported were similar for both ataluren and placebo. ${ }^{86}$

The validity of the clinical study evidence supporting ataluren in DMD is further emphasised by a pre-specified meta-analysis of primary and secondary endpoint data from the ACT-DMD ITT population and the corresponding phase $\mathrm{Ib}$ subgroup. This shows a distinct slowing of disease progression in DMD as a result of ataluren treatment in terms of both 6MWD and TFT parameters (Figure 4). ${ }^{86}$

The above evidence from the literature indicates that when designing clinical studies in DMD, selecting appropriate endpoints is vital and differs according to disease progression for patient population subgroups, the therapy and the duration of treatment. Ataluren is a notable development in DMD management and is a first-in-class agent that addresses the underlying cause of nmDMD in patients with suitable genetic profiles. Individual study results from the phase IIb and phase III studies as well as a pre-specified meta-analysis show a treatment effect for ataluren in nmDMD across primary and secondary endpoints and show that the treatment is well tolerated. 
Figure 4: Meta-analysis of primary and secondary endpoints in phase II and phase III clinical trials of ataluren in Duchenne muscular dystrophy

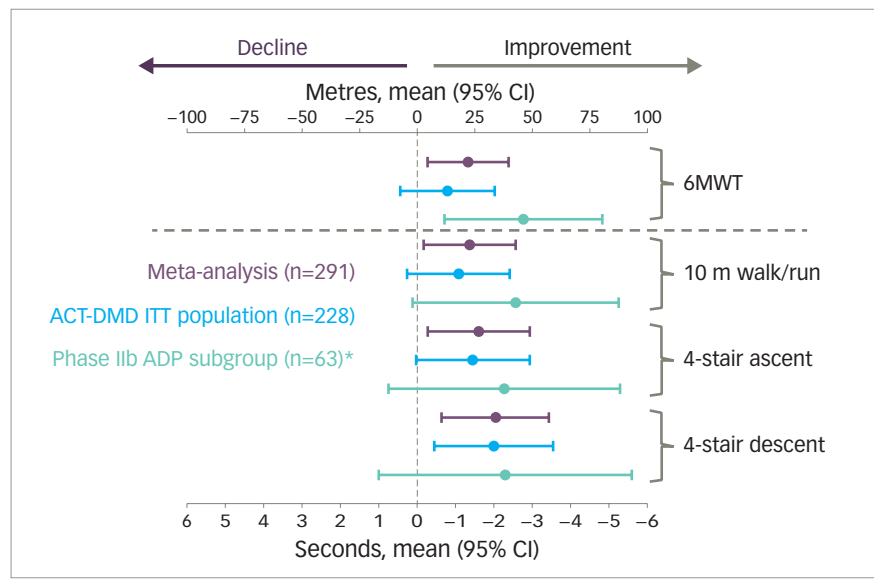

6MWT = 6-minute walk test; $A C T-D M D=$ the phase III ataluren confirmatory trial for Duchenne muscular dystrophy; $A D P=$ ambulatory decline phase; $\mathrm{Cl}=$ confidence interval; ITT = intent to treat. *Including only patients in the ADP subgroup matching ACT-DMD entry criteria. Reproduced with permission from MCDonald et al., 2017.86

\section{Conclusion}

The development of improved genetic techniques such as MLPA and NGS and the recent emergence of disease-modifying drugs have created a turning point in DMD diagnosis and management. Over the past two decades improvement in ventilation at home and the use of corticosteroid treatments has markedly improved life expectancy but new diagnostics and treatments have the potential to still further improve the prognosis. Genetic analysis has largely replaced muscle biopsy techniques and is now considered an essential diagnostic tool since it can identify the differing mutational causes of the disease, identifies carriers, informs genetic counselling and guides treatment choices. Recognition of the urgent need for therapies has led to the development of ataluren and other emerging treatments that enable the partial restoration of dystrophin protein. ${ }^{3,63}$ Such therapeutic approaches may substantially reduce or delay functional decline in patients with DMD, in particular in young boys, at a time in their lives when they would otherwise be active and gaining strength.

In the example case illustrated here, ataluren treatment was beneficial in that it appeared to slow disease progression and prolong ambulation compared to the known natural history. It was effective in combination with corticosteroid treatment and was well tolerated. This case also illustrates the potential benefits of effective treatments and the need to commence them earlier. It is important to recognise that the age of LOA is probably associated with long-term survival and so treatments should aim to maintain patients' ambulation for as long as possible.

In designing clinical trials in DMD it is essential to select an appropriate set of endpoints since no single parameter can capture all of the treatment effects. In the ataluren phase IIb and phase III studies, the use of appropriate endpoints ${ }^{83}$ emphasised the efficacy of the treatment over placebo. In the 48-week study, this treatment efficacy was especially apparent in terms of 6MWD and TFTs where baseline 6MWD was within prescribed limits. This efficacy was further highlighted by the meta-analysis combining data from both studies. In addition, ataluren treatment was well tolerated with a safety profile that was consistent across studies, and showed no marked differences to placebo.

These phase IIb and III studies were of 48-week duration only, which is a relatively short time in which to show profound long-term treatment effects. Data from the extensions of these studies and from real life clinical use will help indicate the long-term value of ataluren and whether its efficacy is compounded in older children who started treatment at an early age.

As the use of ataluren increases and other disease-modifying drugs are introduced, the outlook for children with DMD is likely to improve in coming decades. Realising this improvement will be dependent on awareness of DMD among physicians and a readiness to refer children suspected of having DMD to specialist centres for genetic screening and commencement of treatment as early as possible. $\square$
1. Falzarano MS, Scotton C, Passarelli C, Ferlini A. Duchenne muscular dystrophy: from diagnosis to therapy. Molecules 2015:20:18168-84.

2. Huard J, Mu X, Lu A. Evolving paradigms in clinical pharmacology and therapeutics for the treatment of Duchenne muscular dystrophy. Clin Pharmacol Ther. 2016;100:142-6.

3. Reinig AM, Mirzaei S, Berlau DJ. Advances in the treatment of Duchenne muscular dystrophy: new and emerging pharmacotherapies. Pharmacotherapy. 2017;37:492-9.

4. Strehle EM, Straub V. Recent advances in the management of Duchenne muscular dystrophy. Arch Dis Child. 2015; 100:1173-7.

5. Yiu EM, Kornberg AJ. Duchenne muscular dystrophy. J Paediatr Child Health. 2015;51:759-64

6. Ciafaloni E, Fox DJ, Pandya S, et al. Delayed diagnosis in Duchenne muscular dystrophy: data from the muscular dystrophy surveillance, tracking, and research network (MD dystrophy survellance, tracking, and
STARnet). J Pediatr. 2009:155:380-5.

7. Mohamed K, Appleton R, Nicolaides P. Delayed diagnosis of Duchenne muscular dystrophy. Eur $\mathrm{J}$ Paediatr Neurol. 2000;4:219-23.

8. Bushby K, Finkel R, Birnkrant DJ, et al. Diagnosis and management of Duchenne muscular dystrophy, part 1: diagnosis, and pharmacological and psychosocial management. Lancet Neurol. 2010;9:77-93.

9. Emery AE. Population frequencies of inherited neuromuscular diseases - a world survey. Neuromuscul Disord. 1991:1:19-29.

10. Moat SJ, Bradley DM, Salmon R, et al. Newborn bloodspot screening for Duchenne muscular dystrophy: 21 years experience in Wales (UK). Eur J Hum Genet. 2013;21:1049-53.

11. Mirski KT, Crawford TO. Motor and cognitive delay in Duchenne muscular dystrophy: implication for early diagnosis. $J$ Pediatr. 2014;165:1008-10.

12. Snow WM, Anderson JE, Jakobson LS. Neuropsychological and neurobehavioral functioning in Duchenne muscular dystrophy: a review. Neurosci Biobehav Rev. 2013;37:743-52.

13. Aartsma-Rus A, Ginjaar IB, Bushby K. The importance of genetic diagnosis for Duchenne muscular dystrophy. J Med Genet 2016;53:145-51.

14. Essex C, Roper H. Lesson of the week: late diagnosis of Duchenne's muscular dystrophy presenting as global developmental delay. BMJ. 2001;323:37-8.

15. van Ruiten HJ, Straub V, Bushby K, Guglieri M. Improving recognition of Duchenne muscular dystrophy: a retrospective case note review. Arch Dis Child. 2014;99:1074-7.

16. Eagle M, Baudouin SV, Chandler C, et al. Survival in Duchenne muscular dystrophy: improvements in life expectancy since 1967 and the impact of home nocturnal ventilation. Neuromuscul Disord. 2002;12:926-9.

17. Eagle M, Bourke J, Bullock R, et al. Managing Duchenne muscular dystrophy - the additive effect of spinal surgery and home nocturnal ventilation in improving survival. Neuromuscul Disord. 2007:17:470-5.

18. Bushby K, Finkel R, Birnkrant DJ, et al. Diagnosis and management of Duchenne muscular dystrophy, part 2: implementation of multidisciplinary care. Lancet Neurol. 2010:9:177-89.

19. Matthews $E$, Brassington $R$, Kuntzer $T$, et al. Corticosteroids for the treatment of Duchenne muscular dystrophy. Cochran Database Syst Rev. 2016;5:CD003725.

20. Barber BJ, Andrews JG, Lu Z, et al. Oral corticosteroids and onset of cardiomyopathy in Duchenne muscular dystrophy. J Pediatr. 2013;163:1080-4 e1.

21. Gloss D, Moxley RT, 3rd, Ashwal S, Oskoui M. Practice guideline update summary: corticosteroid treatment of Duchenne muscular dystrophy: report of the guideline development subcommittee of the American Academy of Neurology. Neurology. 2016;86:465-72.

22. Markham LW, Kinnett K, Wong BL, et al. Corticosteroid treatment retards development of ventricular dysfunction in Duchenne muscular dystrophy. Neuromuscul Disord. 2008:18:365-70.

23. McAdam LC, Mayo AL, Alman BA, Biggar WD. The Canadian experience with long-term deflazacort treatment in Duchenne muscular dystrophy. Acta Myol. 2012;31:16-20.
24. Sanzarello I, Merlini L, Traina F, et al. Corticosteroid treatment impact on spinal deformity in Duchenne muscular dystrophy. int Sch Res Notices. 2014:2014:965235.

25. McDonald CM, Henricson EK, Abresch RT, et al. Long-term effects of glucocorticoids on function, quality of life, and survival in patients with Duchenne muscular dystrophy: a prospective cohort study. Lancet. 2018:391:451-61.

26. Kim S, Campbell KA, Fox DJ, et al. Corticosteroid treatments in males with Duchenne muscular dystrophy: treatment duration and time to loss of ambulation. J Child Neurol. 2015;30:1275-80.

27. MCNally EM, Kaltman JR, Benson DW, et al. Contemporary cardiac issues in Duchenne muscular dystrophy. Circulation. 2015;131:1590-8.

28. Duboc $D$, Meune $C$, Lerebours $G$, et al. Effect of perindopril on the onset and progression of left ventricular dysfunction in Duchenne muscular dystrophy. J Am Coll Cardiol. 2005:45:855-7.

29. Duboc $D$, Meune $C$, Pierre $B$, et al. Perindopril preventive treatment on mortality in Duchenne muscular dystrophy: 10 years' follow-up. Am Heart J. 2007;154:596-602.

30. Allen HD, Flanigan KM, Thrush PT, et al. A randomized, double-blind trial of lisinopril and losartan for the treatment of cardiomyopathy in Duchenne muscula dystrophy. PLOS Curr. 2013: DOI: 10.1371/currents. md.2cc69a1dae4be7dfe2bcb420024ea865.

31. Biggar WD, Gingras M, Fehlings DL, et al. Deflazacort treatment of Duchenne muscular dystrophy. J Pediatr. 2001;138:45-50.

32. US Food and Drug Administration. FDA approves drug to treat Duchenne muscular dystrophy. 2017. Available at: www. fda.gov/NewsEvents/Newsroom/PressAnnouncements/ ucm540945.htm (accessed 19 January 2018).

33. Kim S, Zhu Y, Romitti PA, et al. Associations between timing Kim S, Zhu Y, Romitti PA, et al. Associations between timing
of corticosteroid treatment initiation and clinical outcomes in Duchenne muscular dystrophy. Neuromuscul Disord. 2017:27:730-7.

2017;27:730-7

4. Clayton J, Quinlivan R, Marshall J, et al. Extrapolation of 6 -minute walking distance (6MWD) to predict loss of 
ambulation (LOA) with ataluren and placebo in nonsensemutation Duchenne muscular dystrophy (nMDMD). Neuromuscul Disord. 2016;26 (Suppl. 1):S8. P15.

35. Bushby K, Finkel R, Wong B, et al. Ataluren treatment of patients with nonsense mutation dystrophinopathy. Muscle Nerve. 2014;50:477-87.

36. Douglas AG, Wood MJ. Splicing therapy for neuromuscular disease. Mol Cell Neurosci. 2013;56:169-85.

37. Muntoni F, Torelli S, Ferlini A. Dystrophin and mutations: one gene, several proteins, multiple phenotypes Lancet Neurol. 2003;2:731-40.

38. Anthony $\mathrm{K}$, Cirak S, Torelli S, et al. Dystrophin quantification and clinical correlations in Becker muscular dystrophy: implications for clinical trials. Brain. 2011:134:3547-59.

39. Beekman C, Sipkens JA, Testerink J, et al. A sensitive, reproducible and objective immunofluorescence analysis method of dystrophin in individual fibers in samples from patients with Duchenne muscular dystrophy. PLoS One 2014;9:e107494.

40. Aartsma-Rus A, Van Deutekom JC, Fokkema IF, et al. Entries in the Leiden Duchenne muscular dystrophy mutation database: an overview of mutation types and paradoxical cases that confirm the reading-frame rule. Muscle Nerve. 2006:34: $135-44$.

41. Bladen CL, Salgado D, Monges S, et al. The TREAT-NMD DMD Global Database: analysis of more than 7,000 Duchenne muscular dystrophy mutations. Hum Mutat. 2015;36:395-402

42. Tuffery-Giraud S, Béroud C, Leturcq F, et al. Genotypephenotype analysis in 2,405 patients with a dystrophinopathy phenotype analysis in 2,405 patients with a dystrophin
using the UMD-DMD database: a model of nationwide knowledgebase. Hum Mutat. 2009:30:934-45.

43. Monaco AP, Bertelson CJ, Liechti-Gallati S, et al. An explanation for the phenotypic differences between patients bearing partial deletions of the DMD locus. Genomics. 1988;2:90-5.

44. Juan-Mateu J, Gonzalez-Quereda L, Rodriguez MJ, et al. DMD mutations in 576 dystrophinopathy families: a step forward in genotype-phenotype correlations. PLOS One. 2015;10:e0135189

45. Li X, Zhao L, Zhou S, et al. A comprehensive database of Duchenne and Becker muscular dystrophy patients ( $0-18$ years old) in East China. Orphanet J Rare Dis. 2015;10:5.

46. Prior TW, Bridgeman SJ. Experience and strategy for the molecular testing of Duchenne muscular dystrophy. J MOI molecular testing of Duch
Diagn. 2005:7:317-26.

47. Beggs AH, Koenig M, Boyce FM, Kunkel LM. Detection of $98 \%$ of $\mathrm{DMD} / \mathrm{BMD}$ gene deletions by polymerase chain reaction. Hum Genet. 1990;86:45-8.

48. Ishmukhametova A, Khau Van Kien P, Méchin D, et al Comprehensive oligonucleotide array-comparative genomic hybridization analysis: new insights into the molecular pathology of the DMD gene. Eur J Hum Genet. 2012;20:1096-100

49. Manjunath M, Kiran P, Preethish-Kumar V, et al. A comparative study of MPCR, MLPA, and muscle biopsy results in a cohort of children with Duchenne muscular dystrophy: a first study. Neurol India. 2015;63:58-62

50. Nallamilli BR, Ankala A, Hegde M. Molecular diagnosis of Duchenne muscular dystrophy. Curr Protoc Hum Genet. 2014:83:9 25 1-9.

51. Okubo M, Minami N, Goto K, et al. Genetic diagnosis of Duchenne/Becker muscular dystrophy using next-generation sequencing: validation analysis of DMD mutations. J Hum Genet. 2016:61:483-9.

52. Wang Y, Yang Y, Liu J, et al. Whole dystrophin gene analysis by next-generation sequencing: a comprehensive genetic diagnosis of Duchenne and Becker muscular dystrophy. MO Genet Genomics. 2014;289:1013-21.
53. Zimowski JG, Massalska D, Holding M, et al. MLPA based detection of mutations in the dystrophin gene of 180 Polish families with Duchenne/Becker muscular dystrophy. Neuro Neurochir Pol. 2014;48:416-22.

54. Kim MJ, Cho SI, Chae JH, et al. Pitfalls of multiple ligationdependent probe amplifications in detecting DMD exon deletions or duplications. J Mol Diagn. 2016;18:253-9.

55. Santos R, Gonçalves A, Oliveira J, et al. New variants, challenges and pitfalls in DMD genotyping: implications in diagnosis, prognosis and therapy. 1 Hum Genet 2014:59: 454-64.

56. Disset A, Bourgeois CF, Benmalek N, et al. An exon skippingassociated nonsense mutation in the dystrophin gene uncovers a complex interplay between multiple antagonistic splicing elements. Hum Mol Genet. 2006;15:999-1013.

57. Flanigan KM, Dunn DM, von Niederhausern A, et al. Nonsense mutation-associated Becker muscular dystrophy: interplay between exon definition and splicing regulatory elements within the DMD gene. Hum Mutat. 2011;32:299-308.

58. Juan-Mateu J, González-Quereda L, Rodríguez MJ, et al. Interplay between DMD point mutations and splicing signals in dystrophinopathy phenotypes. PLOS One. 2013;8:e59916.

59. Miro J, Laaref AM, Rofidal V, et al. FUBP1: a new protagonist in splicing regulation of the DMD gene. Nucleic Acids Res. 2015;43:2378-89.

60. Tuffery-Giraud S, Miro J, Koenig M, et al. Normal and altered pre-mRNA processing in the DMD gene. Hum Genet. 2017:136:1155-72.

61. Bovolenta M, Neri M, Fini S, et al. A novel custom high density-comparative genomic hybridization array detects common rearrangements as well as deep intronic mutations in dystrophinopathies. BMC Genomics. 2008;9:572.

62. Abbs S, Tuffery-Giraud S, Bakker E, et al. Best practice guidelines on molecular diagnostics in Duchenne/Becker muscular dystrophies. Neuromuscul Disord. 2010;20:422-7.

63. Bello L, Pegoraro E. Genetic diagnosis as a tool for personalized treatment of Duchenne muscular dystrophy. Acta Myol. 2016;35:122-7.

64. Aartsma-Rus A, Krieg AM. FDA approves eteplirsen for Duchenne muscular dystrophy: the next chapter in the eteplirsen saga. Nucleic Acid Ther. 2017:27:1-3.

65. Guiraud S, Chen H, Burns DT, Davies KE. Advances in genetic therapeutic strategies for Duchenne muscular dystrophy. Exp Physiol. 2015:100:1458-67.

66. MCDonald CM, Henricson EK, Han JJ, et al. The 6-minute walk test in Duchenne/Becker muscular dystrophy: Iongitudinal observations. Muscle Nerve. 2010;42:966-74.

67. North Star Clinical Network North Star Ambulatory Assessment. 2011. Available at: www.musculardystrophyuk.org/ assets/0000/6388/NorthStar.pdf (accessed 18 August 2017).

68. Mazzone E, Martinelli D, Berardinelli A, et al. North Star Ambulatory Assessment, 6-minute walk test and timed items in ambulant boys with Duchenne muscular dystrophy. Neuromuscul Disord. 2010;20:712-6.

69. US National Institutes of Health. NCT02310763 - A phase 2 study to evaluate the safety, efficacy, pharmacokinetics and pharmacodynamics of PF-06252616 in Duchenne muscular pharmacodynamics of PF-0625/2616 in Duchenne muscular
dystrophy. Available at: https://clinicaltrials. gov/ct2/show/ NCT02310763 (accessed 18 August 2017).

70. US National Institutes of Heath. NCT02515669 - Study of an investigational drug, BMS-986089, in ambulatory boys with DMD Available at: https://clinicaltrials.gov/ct2/show/ NCT02515669 (accessed 18 August 2017).

71. Mendell JR, Rodino-Klapac LR, Sahenk Z, et al. Eteplirsen for the treatment of Duchenne muscular dystrophy. Ann Neuro. 2013;74:637-47.

72. Nelson MD, Rader F, Tang $X$, et al. PDE5 inhibition alleviates functional muscle ischemia in boys with Duchenne muscular dystrophy. Neurology. 2014;82:2085-91.

73. Voit T, Topaloglu H, Straub V, et al. Safety and efficacy of drisapersen for the treatment of Duchenne muscular dystrophy (DEMAND II): an exploratory, randomised, placebo-controlled phase 2 study. Lancet Neurol. 2014;13:987-96.

74. US National Institutes of Health. NCT02255552 - Confirmatory study of eteplirsen in DMD patients (PROMOVI). Available at: https://clinicaltrials.gov/ct2/show/NCT02255552 (accessed 18 August 2017).

75. Clinical Trials GPS. A randomized double blind, placebo controlled, phase 3 trial of tadalafil for Duchenne muscular dystrophy. Available at: www.clinicaltrialsgps.com/searchclinical-trials/trial-38935/2Location=22434 (accessed 18 August 2017)

76. MCDonald CM, Henricson EK, Abresch RT, et al. The 6-minute walk test and other endpoints in Duchenne muscular dystrophy: longitudinal natural history observations over 48 weeks from a multicenter study. Muscle Nerve. 2013;48:343-56.

77. Sweeney, L. Vandenbone, K. Imaging DMD - developing skeletal muscle MRI/MRS as a biomarker for DMD therapeutic development. Available at: www.parentprojectmd.org/ site/DocServer/Session_8_-_Sweeney_b.pdf?docID=15384 (accessed 18 August 2017).

78. European Medicines Agency. EPAR summary for the public Translarna, ataluren. 2014. Available at: www.ema.europa.eu/ docs/en_GB/document_library/EPAR___Summary_for_the _
public/human/002720/WC500171815.pdf (accessed 18 August 2017).

79. National Institute for Health and Care Excellence (NICE). Press release. NICE recommends ataluren for treating Duchenne muscular dystrophy caused by a nonsense mutation. 2016. Available at: www.nice.org.uk/news/press-and-media/ nice-recommends-ataluren-for-treating-duchenne-musculardystrophy-caused-by-a-nonsense-mutation (accessed 18 August 2017).

80. Agenzia Italiana del Farmaco (AIFA). Translarna: renewal of conditional marketing authorisation. 2016. Available at: www.aifa. gov.it/en/content/translarna-renewal-conditionalmarketing-authorisation (accessed 18 August 2017).

81. Peltz SW, Morsy M, Welch EM, Jacobson A. Ataluren as an agent for therapeutic nonsense suppression. Annu Rev Med. 2013:64:407-25

82. Welch EM, Barton ER, Zhuo J, et al. PTC124 targets genetic disorders caused by nonsense mutations. Nature. 2007:447:87-91

83. Haas $\mathrm{M}$, Vlcek V, Balabanov P, et al. European Medicines Agency review of ataluren for the treatment of ambulant patients aged 5 years and older with Duchenne muscular dystrophy resulting from a nonsense mutation in the dystrophin gene. Neuromuscul Disord. 2015;25:5-13.

84. US Federal Drugs Administration. FDA briefing document. Peripheral and central nervous system drugs advisory committee meeting. NDA 200896 Atatluren. 2017. Available at: www.fda gov/downloads/ AdvisoryCommittees/CommitteesMeetingMaterials/Drugs/ PeripheralandCentralNervousSystemDrugsAdvisoryCommittee UCM577349.pdf (accessed 19 January 2018).

85. MCDonald CM. Use of 6-minute walk distance (6MWD) across Duchenne muscular dystrophy (DMD) studies. Poster presented at: 5th International Congress of Myology, Lyon, France, 14-18 March 2016

86. MCDonald CM, Campbell C, Torricelli RE, et al. Ataluren in patients with nonsense mutation Duchenne muscula dystrophy (ACT DMD): a multicentre, randomised, doubleblind, placebo-controlled, phase 3 trial. Lancet Neurol. 2017;390:1489-98. 